\title{
Tumor neuroendocrino de intestino delgado, con sangrado digestivo oscuro manifiesto: un reporte de caso
}

\section{A case report of a neuroendocrine tumor in the small intestine with manifest dark digestive bleeding}

\author{
Gabriel Mosquera-Klinger, MD, ${ }^{1}$ Juan Esteban Hernández A., MD²
}

\author{
Medicina Interna, Gastroenterología, Hospital \\ "Pablo Tobón Uribe", Medellín, Colombia. Correo \\ electrónico: gami8203@yahoo.com. \\ 2 Médico, Hospital "Pablo Tobón Uribe". Universidad \\ de Antioquia, Medellín, Colombia. Correo \\ electrónico: juanes1027@gmail.com.

\begin{abstract}
Resumen
Presentamos el caso de un paciente joven, sin historia de enfermedad, quien debutó con sangrado digestivo manifiesto por melenas, hematoquecia y anemia severa. Los estudios endoscópicos iniciales fueron normales. En el estudio del intestino delgado con videocápsula endoscópica (VCE) se evidenció una lesión subepitelial ulcerada en el yeyuno, cuyos diagnósticos diferenciales fueron tumor estromal gastrointestinal (GIST) versus tumor neuroendocrino, por lo que se marcó por enteroscopia. Se hicieron estudios de extensión que son normales, por lo que se programó a resección local por laparoscopia haciendo diagnóstico y tratamiento definitivo de un tumor neuroendocrino (TNE).
\end{abstract}

Palabras clave

Hemorragia, intestino delgado, neoplasias del yeyuno, tumor neuroendocrino.

\section{Abstract}

We present the case of a young patient with no history of illness who developed obvious bleeding in the form of melena, hematochezia, and severe anemia. Initial endoscopic studies were normal, but video capsule endoscopy (VCE) revealed an ulcerated subepithelial lesion in the jejunum leading to suspicion of GIST or a neuroendocrine tumor. It was marked in enteroscopy, extension studies showed that it was normal. Therefore, local resection was scheduled by laparoscopy, making diagnosis and management of a neuroendocrine tumor.

Keywords

Hemorrhage, small intestine, neoplasms of the jejunum, neuroendocrine tumor.

\section{INTRODUCCIÓN}

En el $10 \%-20 \%$ de los pacientes con sangrado digestivo no se logra documentar la causa en los estudios endoscópicos iniciales. Para el médico o para el paciente, el sangrado digestivo oscuro puede ser manifiesto, si hay evidencia de hemorragia (melenas, hematoquecia), $\mathrm{u}$ oscuro oculto, en caso de que haya sangre oculta positiva en materia fecal o anemia por deficiencia de hierro inexplicable, sin evidencia clínica de sangrado (1). Debido al crecimiento reciente en los métodos diagnósticos para explorar el intestino delgado, como la videocápsula endoscópica (VCE), la enteroscopia y la angiografía, la mayoría de fuentes de sangrado digestivo en este segmento pueden ser diagnosticadas, por lo que una guía clínica publicada recientemente recomienda el cambio del término sangrado digestivo oscuro a sangrado del intestino delgado (2), y reserva el término sangrado digestivo oscuro para el sangrado que no pueda identificarse después de finalizar toda la evaluación del tracto gastrointestinal (1-3). De todas las causas de sangrado gastrointestinal, solo un pequeño porcentaje ( $5 \%$ ) se atribuye a las fuentes del intestino delgado. En personas jóvenes, las causas pueden ser variadas: desde causas tumorales, como el tumor estromal gastrointestinal (GIST), los tumores neuroendocrinos, los linfomas y los adenocarcinomas, hasta lesiones inflamatorias, en especial por uso de antiinflamatorios no esteroideos 
(AINE) y en enfermedad de Crohn. También se describen causas más raras, como el divertículo de Meckel ulcerado o erosionado y la lesión de Dieulafoy. En nuestro medio, no podemos dejar de pensar en la uncinariasis como causa de anemia y sangrado oscuro (3).

El abordaje inicial del sangrado digestivo proveniente del intestino delgado debe ser con cápsula endoscópica, para establecer la etiología y esclarecer la ubicación, para poder definir el mejor abordaje subsecuente.

\section{CASO CLÍNICO}

Paciente de 26 años, sin antecedentes de enfermedad. Niega consumo de AINE y de tabaquismo e ingiere bebidas alcohólicas de forma esporádica. Ingresa con sangrado digestivo manifiesto por melenas y hematoquecia, con anemia severa. La hemoglobina inicial es de $5,8 \mathrm{~g} / \mathrm{dL}$, por lo que requiere transfusión de dos unidades de glóbulos rojos. Hay sospecha inicial de sangrado digestivo superior. Se realiza esofagogastroduodenoscopia, la cual es normal, sin evidencia de sangrado; luego, una ileocolonoscopia total, que es normal; en esta se evidencia sangre proveniente del intestino delgado. En la VCE se identifica una lesión de aspecto subepitelial, ulcerada, con vaso visible, ubicada en el yeyuno, al $52 \%$ del tránsito de la VCE por el intestino delgado (Figura 1). Por estos hallazgos, se prac- tica una enteroscopia anterógrada de doble balón, con la que se marca el sitio más distal, y se solicita concepto de cirugía para resección local. Se llevan a cabo estudios de estadificación con tomografía axial computarizada (TAC) de tórax y abdomen contrastada, donde no se identifican más lesiones.

Se lleva a laparoscopia, donde se identifica la lesión y se hace resección local oncológica (segmento de $10 \mathrm{~cm}$ ). El paciente presenta una evolución clínica satisfactoria, sin nuevos episodios de sangrado y se le da egreso dos días después. En la actualidad, está asintomático.

\section{DISCUSIÓN}

El sangrado digestivo oscuro manifiesto es un gran reto para el clínico. En el caso descrito, nos adherimos a las recomendaciones actuales de las guías de la Sociedad Estadounidense de Endoscopia Gastrointestinal (ASGE) (en el estudio del sangrado proveniente del intestino delgado) (1). En lo referido a los pacientes con sangrado oscuro manifiesto y estables, se debería hacer el abordaje inicial con VCE, con la finalidad de determinar la localización y el tipo de lesión, para conocer el método y la vía más adecuada de abordaje. En este caso, documentamos una lesión subepitelial ulcerada en el yeyuno, teniendo en cuenta que las causas más comunes de estas lesiones en este grupo de edad son el GIST y el
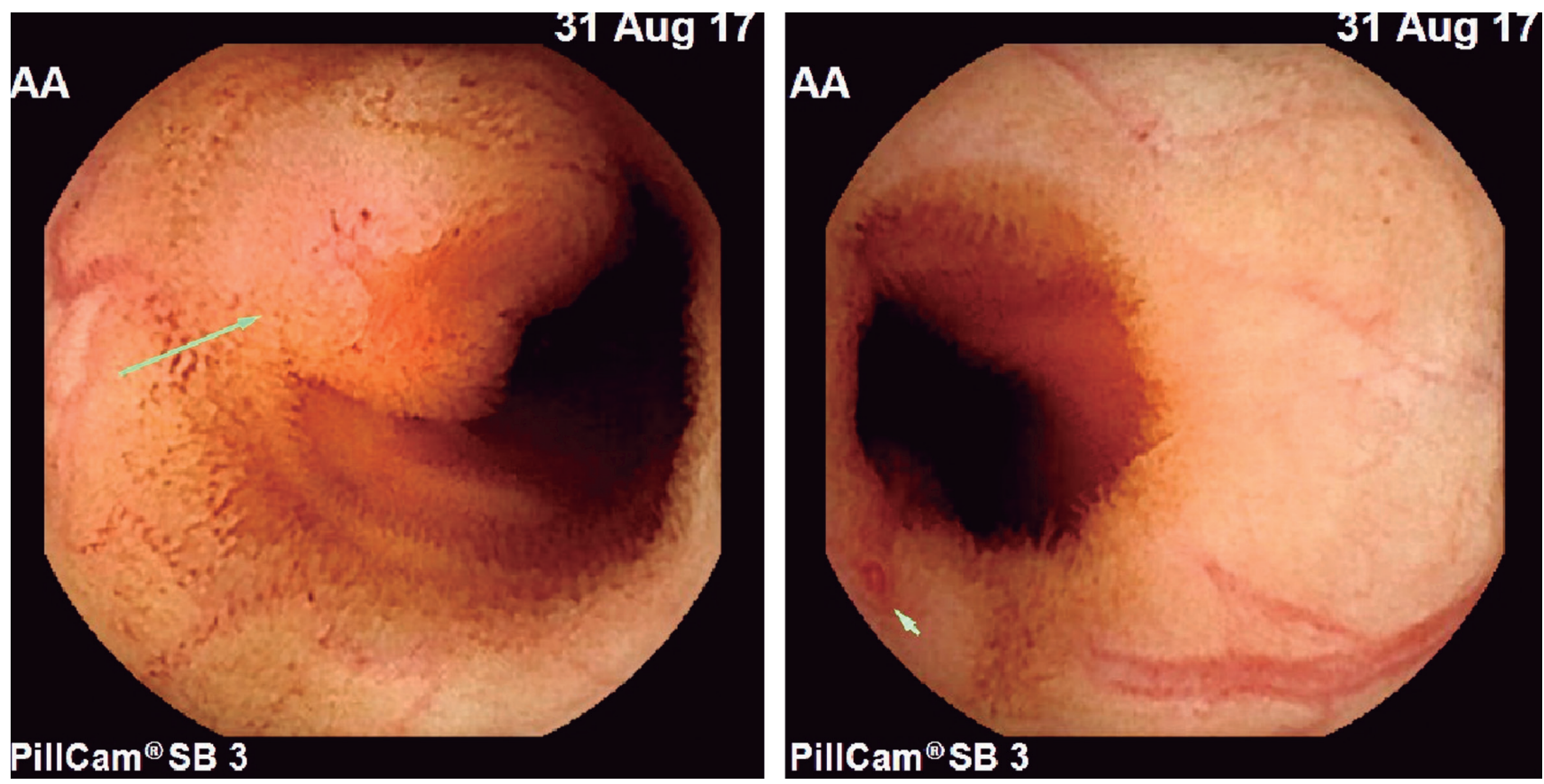

Figura 1. Videocápsula endoscópica que muestra una lesión subepitelial ulcerada en el yeyuno, con vaso visible. 
tumor neuroendocrino. Se realizaron estudios de extensión para definir la resecabilidad. Ante la evidencia de estudios negativos, consideramos llevarlo a laparoscopia y resección local (Figura 2), previa marcación con tinta china mediante enteroscopia de doble balón.

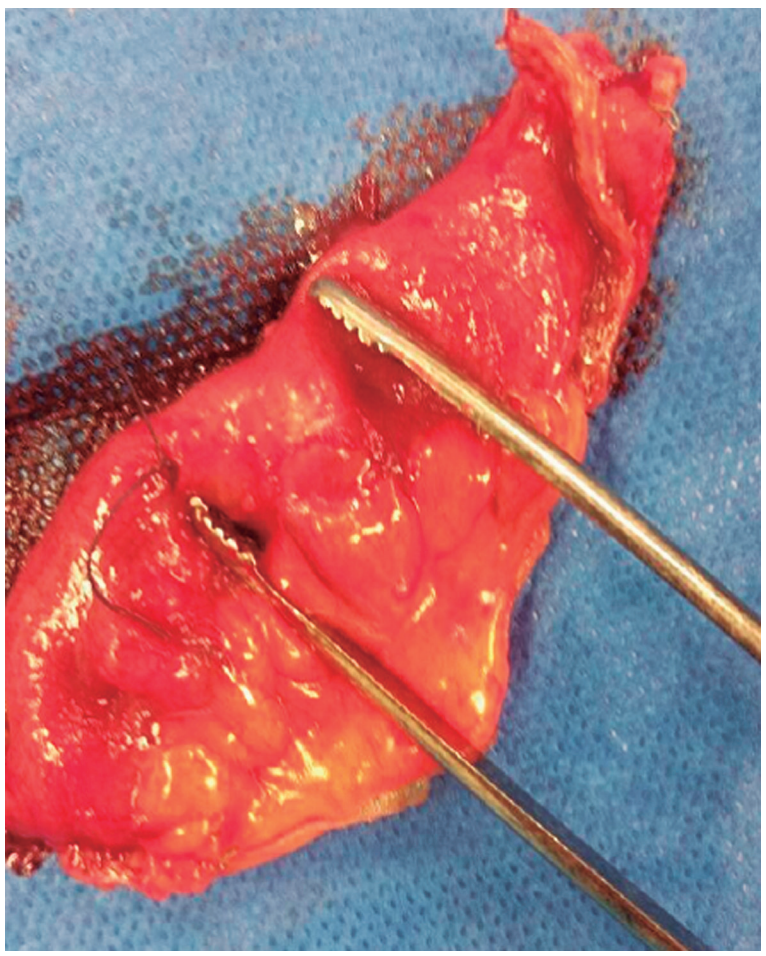

Figura 2. Segmento intestinal resecado.

En la actualidad, disponemos de múltiples técnicas para la evaluación del intestino delgado. Estas incluyen: VCE, enteroscopia de doble balón, enterografía por TAC multifásica (entero-TAC), enterografía por resonancia magnética (entero-RM) y, en raras ocasiones, enteroscopia intraoperatoria. Estas modalidades pueden reconocer las lesiones del intestino delgado y pueden impactar en las estrategias terapéuticas, a menudo evitando intervenciones quirúrgicas innecesarias o disminuyendo los tiempos quirúrgicos por una mejor localización de la lesión. A pesar de estos avances, el enfoque más rentable para el manejo de pacientes con sospecha de sangrado de intestino delgado no ha sido totalmente determinado, pero la recomendación más fuerte en la actualidad es abordar mediante VCE (1, 3-5).

En el caso de los tumores neuroendocrinos en el intestino delgado, un gran número de autores propone usar un enfoque multiimagen en el prequirúrgico (mediante imágenes transversales o funcionales para definir la extensión de la enfermedad). En este sentido, una vez se haya hecho el diagnóstico de tumor en el intestino delgado, la TAC o la reso- nancia magnética (RM) son las modalidades más utilizadas durante el enfoque preoperatorio (6-8). El valor de cada herramienta de diagnóstico varía. La TAC suele ser mejor en la identificación de la lesión primaria, y la RM parece ser mejor en la evaluación de las metástasis hepáticas. Sin embargo, hasta un $15 \%$ de las lesiones podrían no evidenciarse en estos estudios, por lo que podría subestimarse la extensión de la enfermedad metastásica intraabdominal (7, 8). La entero-TAC se ha convertido en una nueva modalidad prometedora en la evaluación del sangrado del intestino delgado (en esta técnica se utiliza el contraste oral neutro de gran volumen para distender el intestino delgado y así mejorar la evaluación de la pared). Además, se administra material de contraste por vía intravenosa y las imágenes se adquieren típicamente en la fase arterial (generalmente, 30 segundos después del bolo intravenoso), fase entérica (50 segundos después del bolo) y fase retardada ( 90 segundos después del bolo) (9). Esta técnica puede detectar lesiones inflamatorias, neoplasias y diferentes lesiones vasculares (angiectasias, várices, lesiones de Dieulafoy, fístulas aortoentéricas y pseudoaneurismas) $(1,9)$. De manera similar a la VCE, la entero-TAC puede ayudar al clínico a determinar si el abordaje anterógrado o retrógrado es más apropiado para realizar la enteroscopia. Incluso, en un estudio publicado por Hakim y colaboradores, se encontraron unas mayores tasas de detección de tumores del intestino delgado con la entero-TAC, comparada con la VCE (10). La entero-TAC, entonces, podría ser considerada como una opción en casos de sangrado digestivo proveniente del intestino delgado en pacientes jóvenes, en quienes la etiología neoplásica se tenga en mente como diagnóstico diferencial.

En un estudio que incluyó 52 pacientes con sangrado digestivo proveniente del intestino delgado con VCE no diagnóstica, se encontró un rendimiento diagnóstico del $50 \%$ con la entero-TAC (11). En otro estudio, que incluyó 30 pacientes con resultados negativos de entero-TAC, se realizó posteriormente VCE y reveló un rendimiento diagnóstico del $57 \%$ (12). Estos datos apoyan el papel complementario de la VCE y de la entero-TAC cuando una de estas es negativa, lo que suma un segundo examen al rendimiento diagnóstico.

La entero-RM podría ser una alternativa cuando haya sospecha de obstrucción en el intestino delgado o en pacientes jóvenes que requieran seguimiento periódico, como en los casos de poliposis o enfermedad de Crohn, para disminuir la exposición a las radiaciones que genera la entero-TAC, aunque, hasta ahora, los datos relacionados con la enteroRM son limitados $(1,13,14)$. En todos los casos de estudio de sangrado digestivo oscuro, se deben tener en cuenta las características individuales de los pacientes, así como los recursos disponibles y la experiencia en la institución con 
los diferentes métodos diagnósticos, para tener un abordaje más racional y efectivo.

En nuestro caso, ante la documentación de una lesión subepitelial en el yeyuno, con vaso visible en dicha localización y por la sospecha de lesión maligna, definimos el tratamiento quirúrgico. El reporte de patología muestra lesión de $15 \times 10 \times 5 \mathrm{~mm}$, unifocal, compatible con tumor neuroendocrino bien diferenciado. El tumor compromete profundamente la subserosa, sin llegar a la serosa, y tiene márgenes negativos (marcadores de sinaptofisina, cromogranina, CD56 con positividad fuerte y difusa en la célula neoplásica, con índice de proliferación Ki-67 bajo en $1 \%$ ) (Figura 3).

Se describe que los tumores neuroendocrinos con localización en el duodeno o el yeyuno proximal son los más raros en el intestino delgado. Se estima que su prevalencia está entre el 5,7 \% y el 7,9\% $(15,16)$. Todos son considerados malignos, excepto los paragangliomas gangliocíticos. La mayoría de los pacientes descritos con este tipo de tumores en el intestino delgado tienen enfermedad metastásica o lesiones múltiples cuando se hace el diagnóstico. En este caso que presentamos, según la clasificación de tumores neuroendocrinos, corresponde al grado 1, que tiene un excelente pronóstico oncológico y bajo riesgo de recaídas $(1,17)$. Recomendamos ver la Tabla 1, donde hacemos un resumen de las clasificaciones de los tumores neuroendocrinos y de la propuesta reciente para clasificar al carcinoma neuroendocrino $(9,18,19)$.

\section{Financiación}

Financiación de recursos propios.
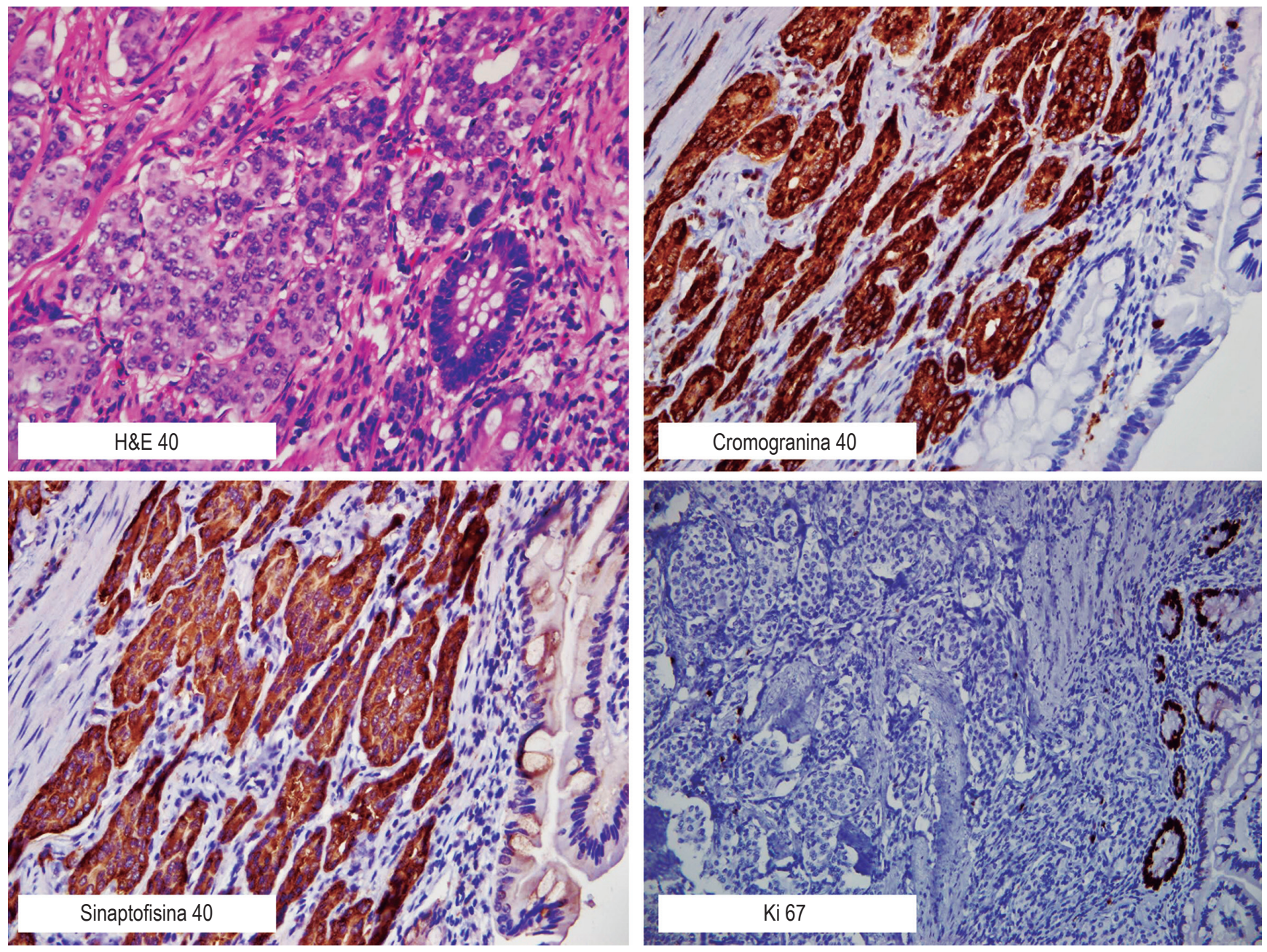

Figura 3. Patología de tumor neuroendocrino, con diferentes marcadores positivos. 
Tabla 1. Clasificación de la OMS de tumores neuroendocrinos gastroenteropancreáticos (9)

\begin{tabular}{|c|c|c|c|}
\hline $\begin{array}{l}\text { Clasificación OMS } 2000 \\
\text { (páncreas) }\end{array}$ & \multicolumn{2}{|c|}{ Invasión local } & Características \\
\hline $\begin{array}{l}\text { Tumor endocrino bien diferenciado, } \\
\text { benigno }\end{array}$ & \multicolumn{2}{|l|}{ Confinado } & $\begin{array}{l}<2 \mathrm{~cm} \text {, no ILV o IPN, <2 mitosis/10 HPF, índice de } \\
\text { proliferación Ki } 67<2 \%\end{array}$ \\
\hline $\begin{array}{l}\text { Tumor endocrino bien diferenciado, } \\
\text { comportamiento incierto }\end{array}$ & \multicolumn{2}{|l|}{ Confinado } & $\begin{array}{l}\text { Uno o más: } \geq 2 \mathrm{~cm} \text {, ILV, IPN, } 2-10 \text { mitosis/10 HPF, } \\
\text { índice de proliferación Ki } 67 \geq 2 \%\end{array}$ \\
\hline $\begin{array}{l}\text { Carcinoma endocrino bien } \\
\text { diferenciado }\end{array}$ & \multicolumn{2}{|l|}{ Invasión local o metástasis } & $\begin{array}{l}\text { Uno o más: } \geq 2 \mathrm{~cm} \text {, ILV, IPN, 2-10 mitosis/10 HPF, } \\
\text { índice de proliferación Ki } 67 \geq 2 \%\end{array}$ \\
\hline $\begin{array}{l}\text { Carcinoma endocrino pobremente } \\
\text { diferenciado }\end{array}$ & \multicolumn{2}{|c|}{ Ampliamente invasivo o metastásico } & Carcinoma de alto grado, con $>10$ mitosis/HPF \\
\hline Clasificación OMS 2010 & \multicolumn{2}{|l|}{ Conteo mitótico } & Índice de proliferación Ki 67 (\%) \\
\hline NET grado 1 & \multicolumn{2}{|l|}{$<2$} & $<3$ \\
\hline NET grado 2 & \multicolumn{2}{|l|}{$2-20$} & $3-20$ \\
\hline NEC & \multicolumn{2}{|l|}{$>20$} & $>20$ \\
\hline \multicolumn{4}{|c|}{ Clasificación OMS 2017 (cambia la clasificación de NEC a WD-NET G3 y PD-NEC) $(9,11,12)$} \\
\hline \multicolumn{4}{|c|}{$\begin{array}{l}\text { Características de alto grado en una neoplasia neuroendocrina (necrosis tumoral, actividad mitótica incrementada >20/10 HPF, índice Ki } 67 \text { alto } \\
>20 \%)\end{array}$} \\
\hline Clasificación & $\begin{array}{c}\text { Examen minucioso del } \\
\text { material patológico }\end{array}$ & $\begin{array}{c}\text { Estudios } \\
\text { inmunohistoquímicos }\end{array}$ & Información clínica \\
\hline WD-NET grado 3 & $\begin{array}{l}\text { Un componente de bajo } \\
\text { grado (OMS G1-G2) } \\
\text { WD-NET; grado inferior } \\
\text { anterior en otra muestra }\end{array}$ & $\begin{array}{l}\text { Pérdida de expresión } \\
\text { de DAXX o ATRX }\end{array}$ & $\begin{array}{l}\text { Hallazgo incidental sin síntomas asociados de } \\
\text { malignidad de alto grado } \\
\text { Marcadores neuroendocrinos elevados en plasma } \\
\text { (cromogranina A) } \\
\text { Avidez difusa en la gamma grafía con octreotida }\end{array}$ \\
\hline PD-NEC & $\begin{array}{l}\text { Un componente de } \\
\text { adenocarcinoma } \\
\text { convencional o carcinoma } \\
\text { de células escamosas }\end{array}$ & $\begin{array}{l}\text { Pérdida de } \mathrm{Rb} \text { o } \\
\text { expresión anormal de } \\
\text { p53 }\end{array}$ & $\begin{array}{l}\text { Síntomas asociados con malignidad de alto grado } \\
\text { Marcadores elevados de carcinoma en plasma (CEA, } \\
\text { CA19.9, CA 125ect) } \\
\text { Actividad negativa o débil y focal en la gammagrafía } \\
\text { con octreotida }\end{array}$ \\
\hline
\end{tabular}

GEP-NET: tumores neuroendocrinos gastroenteropancreáticos; HPF: campos de alto poder; ILV: invasión linfovascular; IPN: invasión perineural; NEC: carcinoma neuroendocrino; OMS: Organización Mundial de la Salud; PD-NEC: carcinoma neuroendocrino pobremente diferenciado; WDNET: tumor neuroendocrino bien diferenciado.

\section{REFERENCIAS}

1. ASGE Standards of Practice Committee, Gurudu S, Bruining DH, Acosta RD, Eloubeidi MA, Faulx AL, et al. The role of endoscopy in the management of suspected small-bowel bleeding. Gastrointest Endosc. 2017;85(1):2231. doi: 10.1016/j.gie.2016.06.013.

2. Gerson LB, Fidler JL, Cave DR, Leighton JA. ACG clinical guideline: diagnosis and management of small bowel bleeding. Am J Gastroenterol. 2015;110(9):1265-87. doi: 10.1038/ajg.2015.246.

3. Pennazio M, Spada C, Eliakim R, Keuchel M, May A, Mulder CJ, et al. Small-bowel capsule endoscopy and device-assisted enteroscopy for diagnosis and treatment of small-bowel disorders: European Society of Gastrointestinal Endoscopy
(ESGE) Clinical Guideline. Endoscopy. 2015;47(4):35276. doi: 10.1055/s-0034-1391855.

4. Mosquera-Klinger G, Correa NF, Concha Mejía A. Sangrado digestivo oscuro y anemia crónica severa: discusión sobre dos causas gastrointestinales subvaloradas en Colombia. Univ Med. 2014;55(2):229-34.

5. Enns RA, Hookey L, Armstrong D, Bernstein CN, Heitman SJ, Teshima C, et al. Clinical practice guidelines for the use of video capsule endoscopy. Gastroenterology. 2017;152(3):497-514. doi: 10.1053/j.gastro.2016.12.032.

6. Chambers AJ, Pasieka JL, Dixon E, Rorstad O. Role of imaging in the preoperative staging of small bowel neuroendocrine tumors. J Am Coll Surg. 2010;211(5):620-7. doi: 10.1016/j.jamcollsurg.2010.07.016.

7. Ahdaleh FS, Lorenzen A, Rajput M, Carr JC, Liao J, Menda $\mathrm{Y}$, et al. The value of preoperative imaging in small bowel neu- 
roendocrine tumors. Ann Surg Oncol. 2013;20(6):1912-7. doi: 10.1245/s10434-012-2836-y.

8. Manguso N, Gangi A, Johnson J, Harit A, Nissen N, Jamil L, et al. The role of pre-operative imaging and double balloon enteroscopy in the surgical management of small bowel neuroendocrine tumors: is it necessary? J Surg Oncol. 2018;117(2):207-12. doi: 10.1002/jso.24825.

9. Huprich JE, Barkow JM, Hansel SL, Alexander JA, Fidler JL. Multiphase CT enterography evaluation of small-bowel vascular lesions. AJR Am J Roentgenol. 2013;201(1):65-72. doi: 10.2214/AJR.12.10414.

10. Hakim FA, Alexander JA, Huprich JE, Grover M, Enders FT. CT-enterography may identify small bowel tumors not detected by capsule endoscopy: eight years experience at Mayo Clinic Rochester. Dig Dis Sci. 2011;56(10):2914-9. doi: 10.1007/s10620-011-1773-0.

11. Agrawal JR, Travis AC, Mortele KJ, Silverman SG, Maurer $\mathrm{R}$, Reddy SI, et al. Diagnostic yield of dual-phase computed tomography enterography in patients with obscure gastrointestinal bleeding and a non-diagnostic capsule endoscopy. J Gastroenterol Hepatol. 2012;27(4):751-9. doi: 10.1111/j.1440-1746.2011.06959.x.

12. Heo HM, Park CH, Lim JS, Lee JH, Kim BK, Cheon JH, et al. The role of capsule endoscopy after negative CT enterography in patients with obscure gastrointestinal bleeding. Eur Radiol 2012;22(6):1159-66. doi: 10.1007/s00330011-2374-1.

13. Wiarda BM, Heine DG, Mensink P, Stolk M, Dees J, Hazenberg HJ, et al. Comparison of magnetic resonance enteroclysis and capsule endoscopy with balloon-assisted enteroscopy in patients with obscure gastrointestinal bleeding. Endoscopy. 2012;44(7):668-73. doi: 10.1055/s0032-1309386.
14. Böcker U, Dinter D, Litterer C, Hummel F, Knebel P, Franke A, et al. Comparison of magnetic resonance imaging and video capsule enteroscopy in diagnosing smallbowel pathology: localization-dependent diagnostic yield. Scand J Gastroenterol. 2010;45(4):490-500. doi: 10.3109/00365520903567817.

15. Vinik AI, McLeod MK, Fig LM, Shapiro B, Lloyd RV, Cho K. Clinical features, diagnosis, and localization of carcinoid tumors and their management. Gastroenterol Clin N Am. 1989;18(4):865-96.

16. Kim JY, Hong SM, Ro JY. Recent updates on grading and classification of neuroendocrine tumors. Ann Diagn Pathol. 2017;29:11-36. doi: 10.1016/j.anndiagpath.2017.04.005.

17. Yin XN, Shen CY, Yin YQ Chen HJ, Chen HN, Yin Y, et al. Prognoses in patients with primary gastrointestinal neuroendocrine neoplasms based on the proposed new classification scheme. Asia Pac J Clin Oncol. 2017;14(2):e37-344. doi: 10.1111/ajco.12760.

18. Tang L, Basturk O, Sue JJ, Klimstra DS. A Practical Approach to the Classification of WHO Grade 3 (G3) Well differentiated Neuroendocrine Tumor (WD-NET) and Poorly Differentiated Neuroendocrine Carcinoma (PD-NEC) of the Pancreas. Am J Surg Pathol. 2016;40(9):1192-202. doi: 10.1097/PAS.0000000000000662.

19. Basturk O, Yang Z, Tang LH, Hruban RH, Adsay V, McCall CM, et al. The High-grade (WHO G3) Pancreatic Neuroendocrine Tumor Category Is Morphologically and Biologically Heterogenous and Includes Both Well Differentiated and Poorly Differentiated Neoplasms. Am J Surg Pathol. 2015;39(5):683-90. doi: 10.1097/ PAS.0000000000000408. 